\title{
Risk Assessment of Typhoon Storm Surge Disasters in Guangdong Province Based on the Improved Fuzzy Bayesian Network
}

\author{
Chengzu Bai ${ }^{1}$, Ren Zhang ${ }^{1}$, Jianqi Zhang ${ }^{2}$, Ting Wang ${ }^{1}$ \\ 1 Research Center of Ocean Environment Numerical Simulation, Institute of Meteorology and \\ Oceanography, PLA of Science and Technology, Nanjing 211101, China \\ 2 Air force of training base of meteorology, Hengyang 421002.China
}

\section{改进型模糊贝叶斯网络对广东省台风风暴潮 的灾害风险评估 \\ 白成祖 ${ }^{1}$, 张 韧 ${ }^{1}$, 张舰齐 ${ }^{2}$, 王 挺 $^{1}$ \\ 1 解放军理工大学气象海洋学院, 军事海洋环境实验室, 南京 211101, 中国 \\ 2 空军气象训练基地, 衡阳 421002, 中国}

\begin{abstract}
To expand insufficient information resource and express fuzzy uncertainty for risk assessment, a new Bayesian network was proposed in this paper. Information diffusion model and triangle fuzzy values were employed to improve the prior probability, using Monte Carlo method to establish the conditional probability tables with the help of AHP. According to the data of typhoon storm surge disasters, economic, cultural and other characteristics, the zoning map of storm surge risk based on Grid and GIS was realized to study disaster risk, vulnerability and capacity of disaster prevention in Guangdong. It showed the results tallied with the practical situation, which may provide the help to reduce the loss caused by storm surge in China.
\end{abstract}

Keywords: risk assessment, information diffusion, storm surge, Bayesian network

\section{摘要}

引入针对信息不完备和体现风险评估模糊不 确定性的贝叶斯网络, 运用信息扩散和三角模
糊数改进优化先验概率, 结合 AHP 建立了蒙特 卡洛主客观条件概率表赋值算法, 并根据 1970 2005 年广东省部分风暴潮最大增水记 录以及沿海区域社会经济、人文、交通等特点, 建立了基于格网和 GIS 插值技术的台风风暴 潮灾害风险评价模型。通过该模型, 对广东沿 海地区的台风风暴潮灾害危险度、脆弱性和防 灾减灾能力进行了分析, 最后绘制出了广东沿 海台风风暴潮灾害概率风险区划图, 结果与实 际风暴潮风险情况基本吻合, 对于我国其他沿 海省市风暴潮防灾减灾工作具有一定的科学 参考意义。

关键词：风险评估, 信息扩散, 风暴潮, 贝 叶斯网络

\section{1. 引言}

全球气候变化背景下, 极端天气事件和气 象灾害频发, 其中风暴潮灾害是我国发生频率 最高、经济损失最重且危害最大的海洋自然灾 害，2008-2013年间风暴潮导致直接经济损失 约 518 亿元, $4.06 \times 10^{3}$ 万人罹遭灾痛, $1.06 \times 10^{3}$ 千公顷农田受淹 ${ }^{[1]}$ 。而中国沿海作为风暴潮影 响第一承险体, 经济发达、人口稠密, 全国 $70 \%$ 
以上的大城市、 $55 \%$ 的国民经济收入都分布于 此, 如何减轻沿海各省台风暴潮灾害造成的损 失, 是我国学术界和经济、社会及管理界面临 的一个重大课题。减灾则意味着要有资金的投 入, 而资金是有限的, 人们所要探索的目标是 怎样以最少的资金获得最大的安全保障, 这是 防灾减灾工作者最关心的问题。这些工作的基 础就是风险评估。虽然台风预报水平在提高, 沿海地区防潮减灾工程不断完善, 但是台风风 暴潮灾害的危害仍未消除, 相反。随着全球变 暖. 海平面上升, 影响我国的台风风暴潮次数 在不断增多, 尤其是沿海地区人口密度的增加 和经济的迅猛发展, 台风风暴潮灾害对我国沿 海地区造成的损失在不断加重。因此, 进行台 风风暴潮灾害风险研究, 通过科学的方法实现 对研究区域风暴潮灾害的评估与区划, 具有很 强的必要性和现实意义 ${ }^{[2]}$ 。

但是对发生概率小却影响深重的特大台 风暴潮等自然灾害进行风险评估, 历史灾情资 料严重不足的问题在所难免。针对此张俊香等

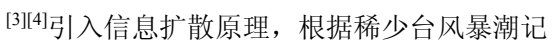
录数据客观计算出广东等中国沿海地区风暴 潮灾害的超越频率, 与实际情况基本吻合, 然 而自然灾害风险往往是致灾因子危险性、承载 体脆弱性相互作用的结果, 因此台风暴潮灾害 风险的评估因子不应局限于潮灾本身; 贝叶斯 网络作为一种诊断概率关系的图形化模型既 可以很好地解决小样本问题 ${ }^{[5-7]}$, 还能依据子 父节点相互关系综合恰当地体现风险灾害和 承载体之间的模糊不确定性, 但其先验概率的 确定往往依据可知概率分布进行假设逼近, 具 有一定主观性。

因此本文综合国内外研究成果, 使用广东 省沿海地区稀疏台风暴潮增水记录及社会经 济、人员交通统计数据, 结合最优信息扩散和 信息分配原理确定先验概率, 以达到提高贝叶 斯网络风险评估的精度, 最后运用GIS格网分 析、插值等技术, 实现了广东省台风风暴潮灾 害危险性、脆弱性、防灾减灾能力以及综合概 率风险区划图的绘制, 可以为广东等沿海地区 防潮减灾, 如灾害人员疏散和救援工作提供科 学决策参考, 也可为保险部门提供一定的技术 支持。

\section{2. 数据与方法}

\section{1 数据来源}

根据风暴潮风险评估的研究方法和分析 要素, 本文采用的数据有: 广东省行政区划图 (2010年), 广东省人口、GDP、通车里程等数 据均来自2005-2010年《广东统计年鉴》, 风 暴潮灾害统计数据从国家海洋局中国海洋公 报中收集整理所得, 风暴潮增水最大记录来自 《台风年鉴》（1970-2005），部分采集自文 献[4, 张俊香, 2008]。

\section{2 贝叶斯网络}

贝叶斯网络是基于贝叶斯定理和条件概 率建立起来的有向无环推理性网络, 网络结构 主要由根结点和非根结点组成, 并以图形化的 方式直观地显示系统内各结点之间的拓补联 系。

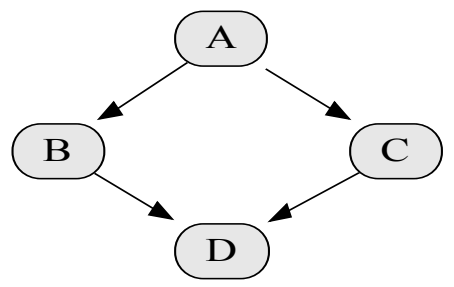

图1.4个结点的简单贝叶斯网络图

Fig.1 Simple Baysian network with 4 nodes

${ }^{[8]}$ 如图1所示, 根节点A依照系统论观点即为最 初 “输入”, 对应的设置参数称作先验概率 $p\left(a_{i}\right), i=1,2, \ldots, \mathrm{n}$, 其余结点B、C、D 统称为非根结点, 对应条件概率 $p\left(b_{j} \mid a_{i}\right)$, $j=1,2, \ldots, \mathrm{m} ; \quad p\left(c_{k} \mid a_{i}\right), k=1,2, \ldots, \mathrm{t}$ $p\left(d_{l} \mid b_{j} c_{k}\right), l=1,2, \ldots, \mathrm{u}$, 则后验概率为: $p\left(d_{l}\right)=\sum_{j=1}^{m} \sum_{k=1}^{t} p\left(d_{l} \mid b_{j} c_{k}\right) p\left(b_{j}\right) p\left(c_{k}\right)$

又据概率公式知:

$p\left(b_{j}\right)=\sum_{i=1}^{n} p\left(b_{j} \mid a_{i}\right)$ 
Risk Analysis and Crisis Response in Big Data Era (RAC-16)

$p\left(c_{k}\right)=\sum_{i=1}^{n} p\left(c_{k} \mid a_{i}\right)$

将式 (2) 代入式 (1) 解得 $p\left(d_{l}\right)$ 。当有更多

节点时，可依上例同理推得。

\section{3 基础数据处理及风险分析流程}

台风暴潮作为发生概率小却危及严重的 特大自然灾害, 对其进行风险评估时, 我们往 往碰到历史灾情资料严重不足的问题 ${ }^{[4]}$ 。在此 种风险认识知识不完备的情况下, 可根据信息 扩散原理, 将传统的观测样本点集值化, 以部 分弥补资料不足的缺陷，达到提高风险估计精 度的目的 ${ }^{[9]}$ 。因此本文首先应用信息扩散模型 对广东沿海稀疏的风暴潮最大增水记录进行 灾害危险性分析, 而后需要开展承灾体的脆弱 性和防减灾能力的调查评估, 鉴于风暴潮自身 复杂的物理机制、数据的不完备性以及风险评 估过程难以避免的一些主观因素, 权衡决定采 用模糊贝叶斯网络整合危险性、脆弱性和防减 灾能力得到既考虑风险不确定性又不失现实 应用性的模糊隶属度综合台风暴潮风险区划, 最后将结果在GIS平台上进行展示为广东省防 灾减灾提供一定的参考意义。整体评估区划的 流程如图2所示。

\section{3. 风险因子分析以及贝叶斯网络建立}

\section{1 危险性计算}

台风风暴潮灾害危险性主要是研究不同 级别台风引起的风暴增水高度, 以及发生频
率。而信息扩散原理可以从稀少不连续的风 暴潮历史数据中推算得到某一增水高度发生 的可能概率, 篇幅所限, 概率计算的具体过 程请参阅文献[4, 张俊香, 2008], 这里仅以汕 尾站点风暴潮统计数据为例说明如何运用信 息扩散原理求得模糊贝叶斯网络中危险性节 点的先验概率。根据文献 [4, 张俊香, 2008] 第六页的描述分析和文献 [10，黄崇福，1995] 中公式（9）－（15）, 得到汕尾风暴潮增水 高度概率分布图（图3），将增水高度划分为 三段论域:

$I_{1}=[0,1.5), I_{2}=[1.5,2.5), I_{3}=[2.5,4]$

然后各段论域进行发生概率积分和标准化处 理 ${ }^{10}$, 将 $I_{1}, I_{2}, I_{3}$ 所得结果 $0.8726,0.1271$, 0.0003 分别设为危险性节点中低风险、中等 风险和高等风险的先验概率。广东省其余区 划单元 (以GIS平台非结构三角形格网作为最 小单元）均依照此例进行危险性分析计算。

\section{2 脆弱性计算}

风暴潮灾害脆弱性用来描述灾害发生时受灾 地区的人口、工业、农作物等对潮灾的遭受 情况, 因此本文选取临海距离、单位面积人 口密度、单位面积工业资产、单位面积主要 农作物种植面积作为脆弱性分析指标（见图 4）。以汕尾人口密度 $\rho_{1}$ 计算为例, 采用三 角模糊数公式进行数据初步处理:

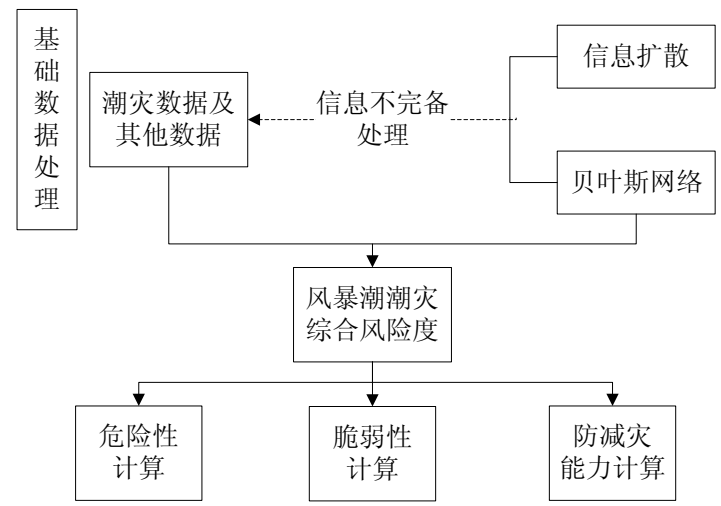

图 2. 台风风暴潮灾害风险评估系统流程图

Fig. 2 Flow chart of risk analyses 


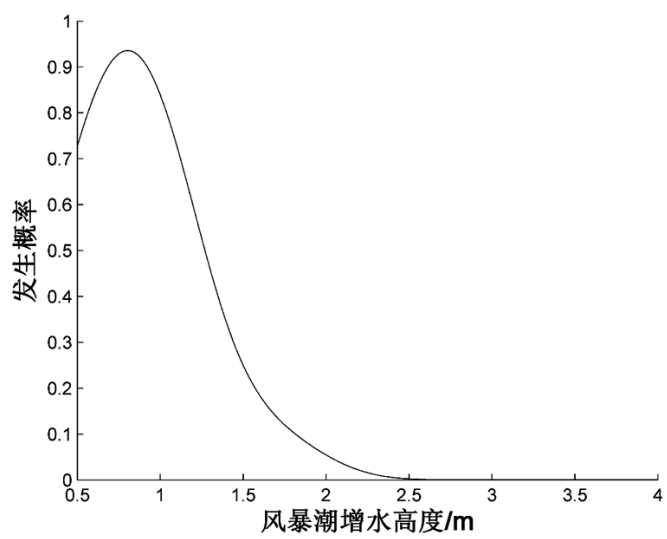

图3. 汕尾风暴潮概率分布图

Fig. 3 The probability of storm surge at Shanwei

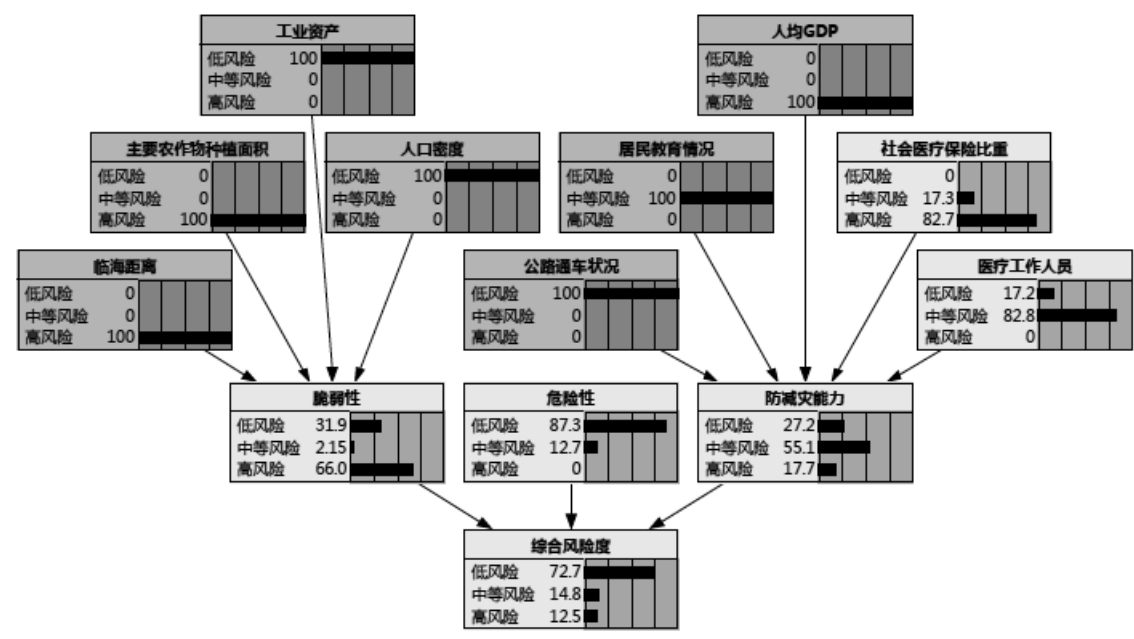

图 4 汕尾风暴潮灾害评估的贝叶斯网络

Fig. 4 The Bayesian network of surge risk assessment at Shanwei

$$
p=\left\{\begin{array}{l}
p_{1}=1-\frac{\left|\rho_{1}-\max (\rho) / 6\right|}{(\max (\rho)-\min (\rho)) / 3} \\
p_{2}=1-\frac{\left|\rho_{1}-\max (\rho) / 2\right|}{(\max (\rho)-\min (\rho)) / 3} \\
p_{3}=1-\frac{\left|\rho_{1}-\max (\rho) \cdot 5 / 6\right|}{(\max (\rho)-\min (\rho)) / 3}
\end{array}\right.
$$

其中 $\rho$ 代表广东省所有区划单元人口密度 的集合。而后对 $p$ 进行标准化处理后 $p_{1}$, $p_{2}, p_{3}$ 即分别为贝叶斯网络中人口密度节 点低风险、中等风险和高等风险的先验概率 输入。

\section{3 防减灾能力计算}

防灾减灾能力指不同区域对风暴潮的规 避与抵御能力, 应与当地的社会人文经济、 救灾部门、交通通讯等要素相关。因此本文 选取人均GDP、医疗人员比重、社会医疗保 险比重、居民教育状况及单位面积公路通车 里程五个主要因素作为防减灾能力衡量指标 
Risk Analysis and Crisis Response in Big Data Era (RAC-16)

（见图4），其计算方法参照脆弱性计算实施。

\section{4 贝叶斯网络条件概率的设置}

确定贝叶斯网络各节点层次关系以及先 验概率赋值之后需要展开讨论的是其非根节 点与其父节点连接的条件概率设置。以脆弱 性条件概率表计算为例, 各个脆弱性指标先 划分为低等风险 (Low), 中等风险 (Median) 和高风险（High）三个等级, 并且由于网络 层次结构复杂，人工一一赋值效率低下，拟 采用蒙特卡洛随机条件概率赋值方法 ${ }^{[11]}$ 。然 而合理的随机赋值必须考虑贝叶斯网络的一 致性以及各节点的重要性排序, 因此本文引 入层次分析法 (Analytic Hierarchy Process, AHP ） ${ }^{[12]}$ 计算各脆弱性指标的权重 $w_{i}$, $i=1,2,3,4$ 。然后在 $[0,0.1]$ 区间随机设立 一基值 $b$, 譬如临海距离（临1）、工业资产 (工3)，人口密度（人4）方面处于低风险, 主要农作物种植面积（主2）处于中等风险, 则对应脆弱性节点所处风险的条件概率为:

$$
p=\left\{\begin{array}{l}
\text { Low }=b_{\text {low }}+w_{1}+w_{2}+w_{4} \\
\text { Median }=b_{\text {median }}+w_{3} \\
\text { High }=b_{\text {high }}
\end{array}\right.
$$

然后对 $p$ 进行标准化处理以达到贝叶斯网 络一致性的要求。由此随机迭代赋基值 10000 次, 最后取平均可得脆弱性节点的风险条件 概率表, 如表1所示。

最后确定了先验概率和条件概率等参数 后, 将其输入贝叶斯网络得到区划单元的综 合风险模糊评估结果, 以汕尾为例进行贝叶 斯网络整体演示 (图4), 由图4可知汕尾面临 风暴潮灾害袭击的低风险模糊隶属度为 $72.7 \%$, 中等风险为 $14.8 \%$, 高风险为 $12.5 \%$ 。

根据第2节方法的描述与计算结果，最后 以GIS平台为支撑, 将原本分辨率较粗的不规 则三角形区划单元风险度值进行插值（本文 采用的是较为成熟先进的Kriging插值算法) 以得出更加精细化的广东省风暴潮灾害风险 区划图（图5）。
表1 脆弱性节点的条件概率表

Table 1 The conditional probability for "Vulnerability"

\begin{tabular}{|c|c|c|c|c|c|c|}
\hline 临 1 & 主 2 & 工 3 & 人 4 & $\mathrm{~L}$ & $\mathrm{M}$ & $\mathrm{H}$ \\
\hline L & L & L & L & 0.96 & 0.02 & 0.02 \\
\hline $\mathrm{L}$ & L & L & $\mathrm{M}$ & 0.42 & 0.54 & 0.04 \\
\hline $\mathrm{L}$ & $\mathrm{L}$ & L & $\mathrm{H}$ & 0.43 & 0.01 & 0.56 \\
\hline $\mathrm{L}$ & $\mathrm{L}$ & $\mathrm{M}$ & L & 0.73 & 0.26 & 0.01 \\
\hline $\mathrm{L}$ & $\mathrm{L}$ & $\mathrm{M}$ & $\mathrm{M}$ & 0.19 & 0.77 & 0.04 \\
\hline $\mathrm{L}$ & $\mathrm{L}$ & $\mathrm{M}$ & $\mathrm{H}$ & 0.18 & 0.26 & 0.56 \\
\hline $\mathrm{L}$ & $\mathrm{L}$ & $\mathrm{H}$ & L & 0.70 & 0.03 & 0.27 \\
\hline $\mathrm{L}$ & $\mathrm{L}$ & $\mathrm{H}$ & $\mathrm{M}$ & 0.18 & 0.55 & 0.27 \\
\hline $\mathrm{L}$ & L & $\mathrm{H}$ & $\mathrm{H}$ & 0.19 & 0.03 & 0.78 \\
\hline $\mathrm{L}$ & M & L & L & 0.94 & 0.06 & 0.00 \\
\hline$\vdots$ & 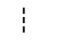 & 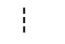 & ! & ! & $\vdots$ & $\vdots$ \\
\hline $\mathrm{L}$ & $\mathrm{M}$ & L & M & 0.37 & 0.59 & 0.04 \\
\hline $\mathrm{L}$ & $\mathrm{M}$ & $\mathrm{L}$ & $\mathrm{H}$ & 0.38 & 0.07 & 0.55 \\
\hline $\mathrm{L}$ & M & $\mathrm{M}$ & $\mathrm{L}$ & 0.64 & 0.32 & 0.04 \\
\hline $\mathrm{L}$ & $\mathrm{M}$ & $\mathrm{M}$ & $\mathrm{M}$ & 0.12 & 0.88 & 0.00 \\
\hline $\mathrm{L}$ & M & $\mathrm{M}$ & $\mathrm{H}$ & 0.13 & 0.32 & 0.55 \\
\hline $\mathrm{H}$ & $\mathrm{H}$ & L & $\mathrm{H}$ & 0.27 & 0.01 & 0.72 \\
\hline $\mathrm{H}$ & $\mathrm{H}$ & $\mathrm{M}$ & $\mathrm{L}$ & 0.56 & 0.26 & 0.17 \\
\hline $\mathrm{H}$ & $\mathrm{H}$ & $\mathrm{M}$ & $\mathrm{M}$ & 0.02 & 0.80 & 0.18 \\
\hline $\mathrm{H}$ & $\mathrm{H}$ & $\mathrm{M}$ & $\mathrm{H}$ & 0.00 & 0.26 & 0.74 \\
\hline $\mathrm{H}$ & $\mathrm{H}$ & $\mathrm{H}$ & L & 0.55 & 0.03 & 0.43 \\
\hline $\mathrm{H}$ & $\mathrm{H}$ & $\mathrm{H}$ & $\mathrm{M}$ & 0.00 & 0.56 & 0.43 \\
\hline $\mathrm{H}$ & $\mathrm{H}$ & $\mathrm{H}$ & $\mathrm{H}$ & 0.03 & 0.03 & 0.94 \\
\hline
\end{tabular}

如图5（a）所示, 粤西沿岸高风险隶属 度最高, 尤其是雷州半岛东段平均高于 $30 \%$, 说明此处台风暴潮灾害威胁可能性最大、受 灾程度最严重, 这与历史灾情数据以及文献 4结果相符。沿海岸线依次向东高风险隶属度 呈下降趋势, 珠江三角洲段台风暴潮灾害的 风险相对最低, 这与当地风暴潮增水高度较 低, 淹没范围较小, 经济实力相对雄厚进而 防范力度较高的现实相符。粤东汕头一带风 险略高，风暴潮灾防范措施应该参照此区划 结果进行相应等级部署, 尽可能地避免财产 和人员损失; 图5（c）低风险隶属度分布与5 （a）结果相符, 即正好呈相反趋势; 图5 (b) 验证加强了 5（a）的计算可信度。 
Risk Analysis and Crisis Response in Big Data Era (RAC-16)

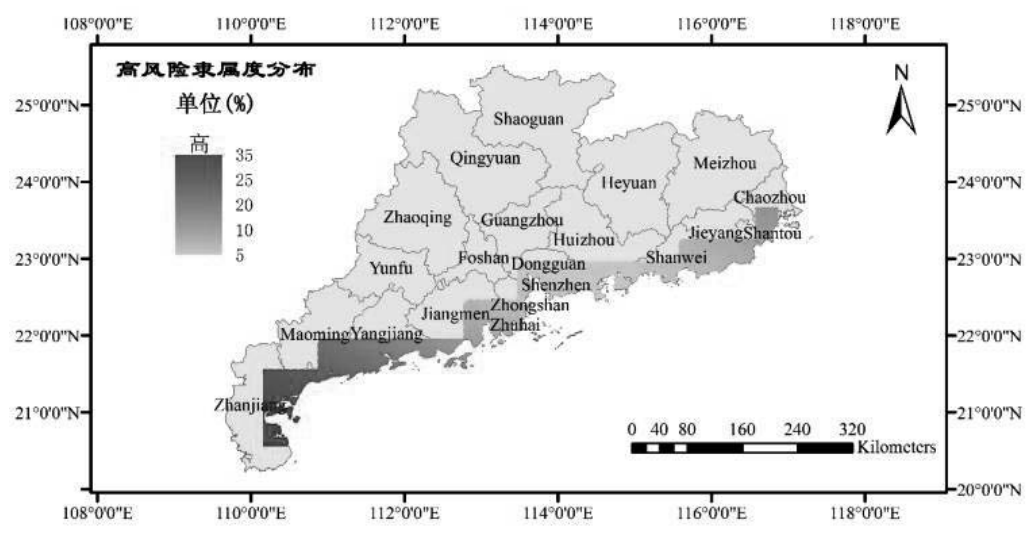

5 (a)

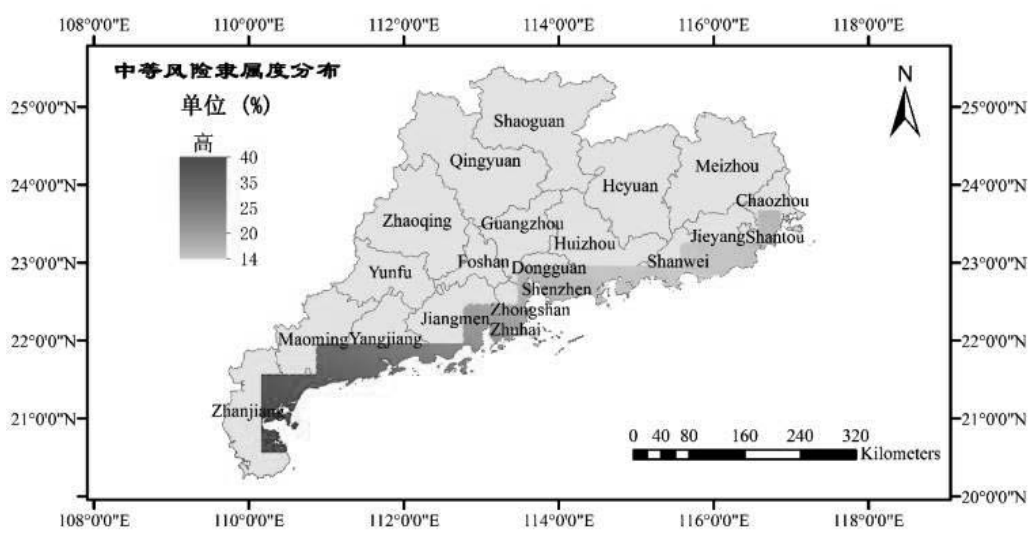

5 (b)

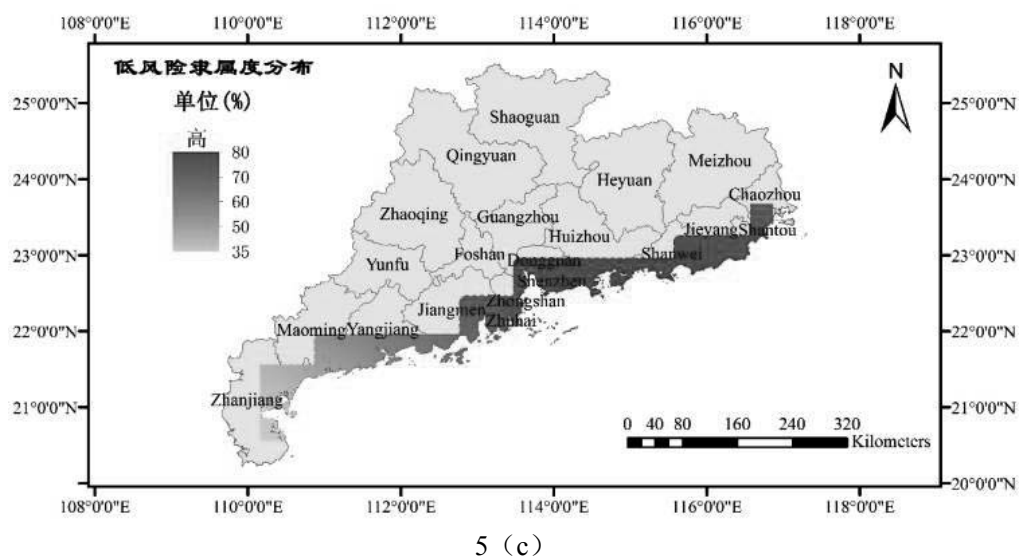

图 5 广东省风暴潮灾害风险区划图

Fig. 5 The zoning map of surge risk assessment in Guangdong 
Risk Analysis and Crisis Response in Big Data Era (RAC-16)

\section{5 结论}

本文依照稀疏的广东省风暴潮最大增水 记录以及相关农业、经济、人文信息数据, 应用信息扩散技术三角模糊数优化、AHP等 方法优化模糊贝叶斯网络, 并采用GIS网格分 析以及插值等技术为支持, 得到比较精细化 的广东省台风风暴潮灾害风险区划图，结果 显示广东省雷州半岛东岸、奥东的汕头一带 等是台风暴潮灾害的频发区和重点防范区。 虽然研究分析中部分风险因素情况不够全 面, 如选取风险分析指标有待商榷, 但相信 随着统计测绘等技术的进步，以及对台风风 暴潮形成机制的进一步研究, 可以更好地提 高对台风风暴潮风险预报和分析的能力, 为 防灾减灾提供更加科学的参考建议。

\section{参考文献}

[1]State Oceanic Administration. China Ocean Bulletin (2008- 2012) [EB /OL]. [2014 - 02 01], http: //www. coi.gov.cn/gongbao/zaihai

[2]Samani B A, Shahbodaghlou F. A fuzzy systematic approach to construction risk analysis. J. Risk Anal. Crisis Response, 2012, 2(4): 275-284

[3] 张俊香, 李平日, 黄光庆等. 基于信息扩 散理论的中国沿海特大台风暴潮灾害风险 分析. 热带地理, 2007, 27(1):11-14

[4]张俊香, 黄崇福, 刘旭拢. 广东沿海台风暴 潮灾害的地理分布特征和风险评估 (1949-2005). 应用基础与工程科学学报, 2008, 16(3):393-402

[5] Pearl J. Causal diagrams for empirical research. Biometrika, 1995, 82:669-710

[6] Liaofa Li, Jinfeng Wang, Hareton Leung, et al. A Bayesian Method to Mine Spatial Data Sets to Evaluate the Vulnerability of Human Beings to Catastrophic Risk. Risk Analysis, 2012

[7] Weihua F. Bayesian theory and robust control strategy in risk management of large-scale engineering project. Management and Service Science, 2009. MASS'09. International Conference on IEEE, 2009:
1-7.

[8] Lampinen J, Vehtari A. Bayesian approach for neural networks--review and cage studies. Neural Networks, 2001, 14:257-274

[9]Huang Chongfu. Information Matrix and Application. Journal of General Systems, 2001, 30(6): 603-622

[10] 黄崇福, 王家鼎. 模糊信息优化处理技术 及其应用. 北京: 航空航天大学出版杜, 1995

[ 11 ] Madigan, D. and York, J. Bayesian graphical models for discrete data. International Statistical Review, 1995, 63: 215-232.

[12]T. L. Saaty. The analytic hierarchy process. MacGraw Hill, New York, 1980. 